\title{
Vertex-partitioning into fixed additive induced-hereditary properties is NP-hard
}

\author{
Alastair Farrugia \\ afarrugia@alumni.uwaterloo.ca \\ Malta
}

Submitted: Dec 19, 2002; Accepted: Jul 12, 2004; Published: Jul 19, 2004

MR Subject Classifications: 05C15 (Primary); 05C85, 68Q17 (Secondary)

\begin{abstract}
Can the vertices of an arbitrary graph $G$ be partitioned into $A \cup B$, so that $G[A]$ is a line-graph and $G[B]$ is a forest? Can $G$ be partitioned into a planar graph and a perfect graph? The NP-completeness of these problems are special cases of our result: if $\mathcal{P}$ and $\mathcal{Q}$ are additive induced-hereditary graph properties, then $(\mathcal{P}, \mathcal{Q})$ colouring is NP-hard, with the sole exception of graph 2-colouring (the case where both $\mathcal{P}$ and $\mathcal{Q}$ are the set $\mathcal{O}$ of finite edgeless graphs). Moreover, $(\mathcal{P}, \mathcal{Q})$-colouring is NP-complete iff $\mathcal{P}$ - and $\mathcal{Q}$-recognition are both in NP. This completes the proof of a conjecture of Kratochvíl and Schiermeyer, various authors having already settled many sub-cases.
\end{abstract}

Kratochvíl and Schiermeyer conjectured in [19] that for any additive hereditary graph properties $\mathcal{P}$ and $\mathcal{Q}$, recognising graphs in $\mathcal{P} \circ \mathcal{Q}$ is NP-hard, with the obvious exception of bipartite graphs (the case where both $\mathcal{P}$ and $\mathcal{Q}$ are the set $\mathcal{O}$ of finite edgeless graphs). They settled the case where $\mathcal{Q}=\mathcal{O}$, and it was natural to extend the conjecture to induced-hereditary properties. Berger's result [3] that reducible additive inducedhereditary properties have infinitely many minimal forbidden subgraphs provided support for the extended conjecture.

We prove the extension of the Kratochvíl-Schiermeyer conjecture in this paper. Problems such as the following (for an arbitrary graph $G$ ) are therefore NP-complete. Can $V(G)$ be partitioned into $A \cup B$, so that $G[A]$ is a line-graph and $G[B]$ is a forest? Can $G$ be partitioned into a planar graph and a perfect graph? For fixed $k, \ell, m$, can $G$ be partitioned into a $k$-degenerate subgraph, a subgraph of maximum degree $\ell$, and an $m$ edge-colourable subgraph?

Garey et al. $[15,22]$ essentially showed $(\mathcal{O},\{$ forests $\})$-colouring to be NP-complete, while Brandstädt et al. [4, Thm. 3] proved the case $\left(\mathcal{O},\left\{P_{4}, C_{4}\right\}\right.$ - free graphs). 
Let $\mathcal{P}$ be a property and let $\mathcal{P}^{k}$ be the product of $\mathcal{P}$ with itself, $k$ times. Brown and Corneil $[6,8]$ showed that $\mathcal{P}^{k}$-recognition is NP-hard when $\mathcal{P}$ is the set of perfect graphs and $k \geq 2$, while Hakimi and Schmeichel [17] did the case \{forests ${ }^{2}$. There was particular interest in $G$-free $k$-colouring (where $\mathcal{P}$ has just one forbidden induced-subgraph $G$ ). When $G=K_{2}$ we get graph colouring, one of the best known NP-complete problems, while subchromatic number $[2,13]$ (partitioning into subgraphs whose components are all cliques) is the case $G=P_{3}$. Brown [7] proved the case where $G$ is 2-connected, and Achlioptas [1] showed NP-completeness for all $G$. In fact, Achlioptas' proof settles the case $\mathcal{R}^{k}$ for any irreducible additive induced-hereditary $\mathcal{R}$.

\section{Preliminaries}

We consider only simple finite graphs, referring to [14] and [25] for general definitions in complexity and graph theory. We write $G \leq H$ when $G$ is an induced subgraph of $H$. We identify a graph property with the set of graphs that have that property. A property $\mathcal{P}$ is additive, or (induced-)hereditary, if it is closed under taking vertex-disjoint unions, or (induced-)subgraphs. The properties we consider contain the null graph $K_{0}$ and at least one, but not all (finite simple non-null) graphs.

A $(\mathcal{P}, \mathcal{Q})$-colouring of $G$ is a partition of $V(G)$ into red and blue vertices, such that the red vertices induce a subgraph $G_{\mathcal{P}} \in \mathcal{P}$, and the blue vertices induce a subgraph $G_{\mathcal{Q}} \in \mathcal{Q}$. The product of $\mathcal{P}$ and $\mathcal{Q}$ is $\mathcal{P} \circ \mathcal{Q}$, the set of $(\mathcal{P}, \mathcal{Q})$-colourable graphs. We use $(\mathcal{P}, \mathcal{Q})$-colouring, $(\mathcal{P}, \mathcal{Q})$-partition and $(\mathcal{P} \circ \mathcal{Q})$-recognition interchangeably.

Let $\mathcal{P}$ be an additive induced-hereditary property. Then $\mathcal{P}$ is reducible if it is the product of two additive induced-hereditary properties; otherwise it is irreducible. It is true, though by no means obvious, that if $\mathcal{P}$ is the product of any two properties, then it is also the product of two additive induced-hereditary properties [11].

Now let $\mathcal{P}$ be any induced-hereditary property. The set of minimal forbidden inducedsubgraphs for $\mathcal{P}$ is $\mathcal{F}(\mathcal{P}):=\{H \notin \mathcal{P} \mid \forall G<H, G \in \mathcal{P}\}$. Note that $\mathcal{F}(\mathcal{O})=\left\{K_{2}\right\}$, while all other induced-hereditary properties have forbidden subgraphs with at least 3 vertices. $\mathcal{P}$ is also additive iff every graph in $\mathcal{F}(\mathcal{P})$ is connected. Every hereditary property is induced-hereditary, and the product of additive (induced-hereditary) properties is additive (induced-hereditary).

A graph $H$ is strongly ${ }^{1}$ uniquely $\left(\mathcal{P}_{1}, \ldots, \mathcal{P}_{n}\right)$-partitionable if there is exactly one ordered partition $\left(V_{1}, \ldots, V_{n}\right)$ of $V(H)$ such that for all $i, H\left[V_{i}\right] \in \mathcal{P}_{i}$. More precisely, suppose $V(H)=U_{1} \cup \cdots \cup U_{n}$, where $H\left[U_{i}\right] \in \mathcal{P}_{i}$ for all $i$. Then there is a permutation $\phi$ of $\{1, \ldots, n\}$ such that, for every $i$ :

(a) $V_{i}=U_{\phi(i)}$;

(b) $P_{i}=P_{\phi(i)}$.

When the $\mathcal{P}_{i}$ 's are additive induced-hereditary and irreducible, Mihók [21] gave a construction that can easily be adapted (cf. [10, Thm. 5.3], [11], [5]) to give a strongly uniquely $\left(\mathcal{P}_{1}, \ldots, \mathcal{P}_{n}\right)$-partitionable graph $H$ with $V_{n} \neq \emptyset$. We use $H$ to show that $\mathcal{A} \circ \mathcal{B}$ -

\footnotetext{
${ }^{1}$ Without condition (b), $H$ would just be uniquely $\left(\mathcal{P}_{1}, \ldots, \mathcal{P}_{n}\right)$-partitionable.
} 
recognition is at least as hard as $\mathcal{A}$-recognition, when $\mathcal{A}$ and $\mathcal{B}$ are additive inducedhereditary properties (the result is not true for all properties, e.g., $\mathcal{B}:=\{G|| V(G) \mid \geq$ $10\})$.

1. Theorem. Let $\mathcal{A}$ and $\mathcal{B}$ be additive induced-hereditary properties. Then there is a polynomial-time transformation from the $\mathcal{A}$-recognition problem to the $(\mathcal{A} \circ \mathcal{B})$-recognition problem.

Proof: It is clearly enough to prove this when $\mathcal{B}$ is irreducible. For any graph $G$ we will construct (in time linear in $|V(G)|$ ) a graph $G^{\prime}$ such that $G \in \mathcal{A}$ if and only if $G^{\prime} \in \mathcal{A} \circ \mathcal{B}$.

Let $\mathcal{A}=\mathcal{P}_{1} \circ \cdots \circ \mathcal{P}_{n-1}, \mathcal{B}=\mathcal{P}_{n}$, where the $\mathcal{P}_{i}$ 's are irreducible additive inducedhereditary properties. Let $H$ be a fixed strongly uniquely $\left(\mathcal{P}_{1}, \ldots, \mathcal{P}_{n}\right)$-partitionable graph, with partition $\left(V_{1}, \ldots, V_{n}\right)$, such that $V_{n} \neq \emptyset$. Let $v_{H}$ be some fixed vertex that is not in $V_{n}$, say $v_{H} \in V_{1}$.

For any graph $G$, we construct $G^{\prime}$ by taking a copy of $G$ and a copy of $H$, and making every vertex of $G$ adjacent to every vertex of $N\left(v_{H}\right) \cap V_{n}$. By additivity of $\mathcal{A}$, if $G$ is in $\mathcal{A}$, then $G^{\prime}$ is in $\mathcal{A} \circ \mathcal{B}$.

Conversely, if $G^{\prime} \in \mathcal{A} \circ \mathcal{B}=\mathcal{P}_{1} \circ \cdots \circ \mathcal{P}_{n}$, then it has an ordered partition $\left(W_{1}, \ldots, W_{n}\right)$ with $W_{i} \in \mathcal{P}_{i}$ for each $i$. Since the $\mathcal{P}_{i}$ 's are induced-hereditary, $G^{\prime}\left[W_{i}\right] \in \mathcal{P}_{i}$ implies $G^{\prime}\left[W_{i} \cap V(H)\right] \in \mathcal{P}_{i}$. $\operatorname{Then}^{2}\left(W_{1} \cap V(H), \ldots, W_{n} \cap V(H)\right)=\left(V_{1}, \ldots, V_{n}\right)$; in particular, $v_{H} \in W_{1}$.

Suppose some $z \in V(G)$ is in $W_{n}$. Now $\left(V_{1} \backslash\left\{v_{H}\right\}, V_{2}, \ldots, V_{n-1}, V_{n} \cup\{z\}\right)$ is a $\left(\mathcal{P}_{1}, \ldots, \mathcal{P}_{n}\right)$-partition of $\left(H-v_{H}\right)+z \cong H$. Then $\left(V_{1} \backslash\left\{v_{H}\right\}, V_{2}, \ldots, V_{n-1}, V_{n} \cup\left\{v_{H}\right\}\right)$ is a $\left(\mathcal{P}_{1}, \ldots, \mathcal{P}_{n}\right)$-partition of $H$ that is different from $\left(V_{1}, \ldots, V_{n}\right)$ (since $V_{n} \neq \emptyset$ ), a contradiction.

Thus no vertex of $G$ is in $W_{n}$, and so $G \leq G^{\prime}\left[W_{1} \cup \cdots \cup W_{n-1}\right] \in \mathcal{P}_{1} \circ \cdots \circ \mathcal{P}_{n-1}=\mathcal{A}$, and $G \in \mathcal{A}$ as required.

\section{NP-hardness}

We will prove the main result by transforming a version of $p$-IN- $r$-SAT to $(\mathcal{P}, \mathcal{Q})$-colouring, where $p$ and $r$ are fixed integers depending on $\mathcal{P}$ and $\mathcal{Q}$. We recall that $p$-IN- $r$-SAT is the problem of determining whether an arbitrary formula with clauses of size $r$ has a valid truth assignment that sets exactly $p$ literals to TRUE in each clause? Schaefer [24] showed this to be NP-complete even for formulae with all literals unnegated, for any fixed $p$ and $r$, so long as $1 \leq p<r$ and $r \geq 3$. We restate this version as:

$p$-IN- $r$-COLOURING

Instance: an $r$-uniform hypergraph.

Problem: is there a set of vertices $U$ such that, for each hyper-edge $e,|U \cap e|=p$ ?

\footnotetext{
${ }^{2} \mathrm{Up}$ to some permutation of the subscripts as in (a), (b).
} 


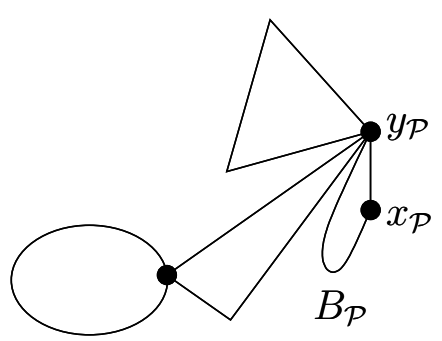

$F_{\mathcal{P}}$

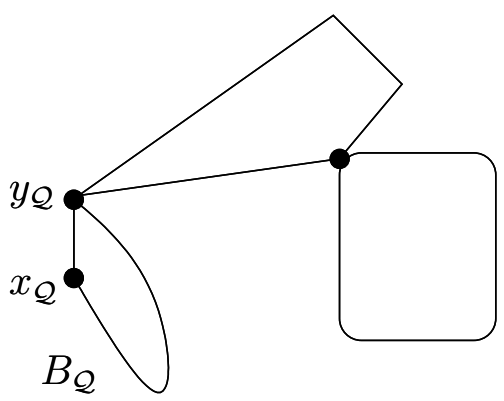

$F_{\mathcal{Q}}$

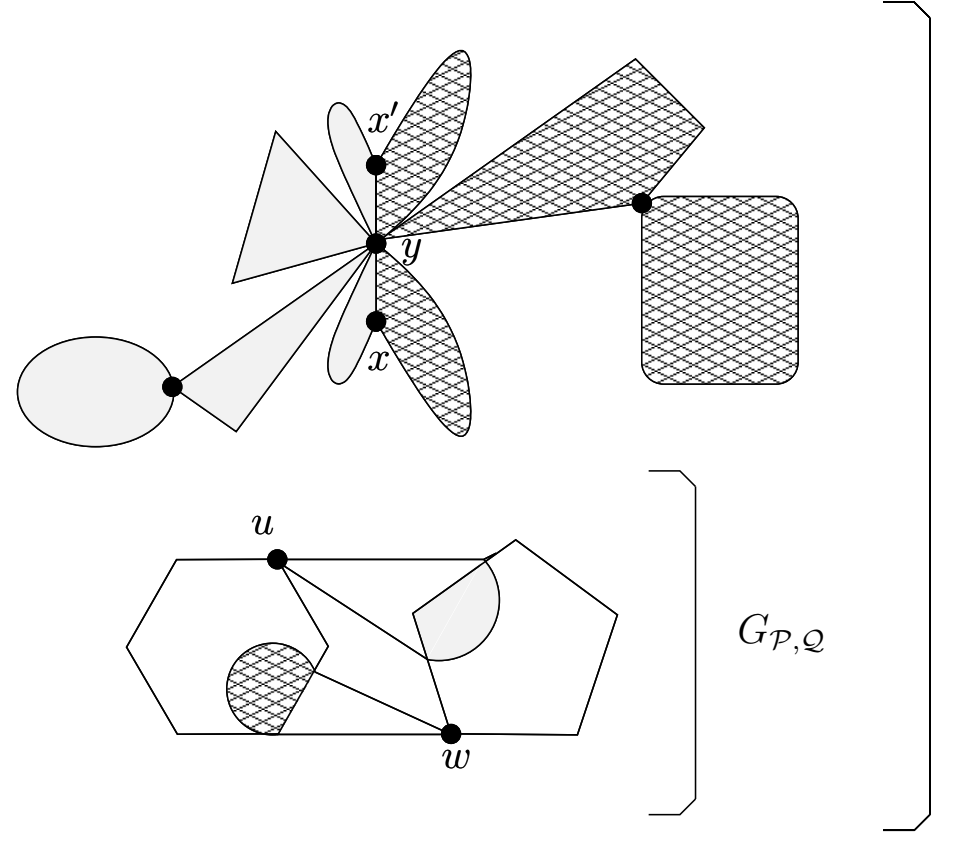

Figure 1: The forbidden graphs $F_{\mathcal{P}}$ and $F_{\mathcal{Q}}$, and the replicator gadget $R$. The shaded neighbours of $u$ in $G_{\mathcal{P}, \mathcal{Q}}$ are connected to the other shaded vertices in $R$. The hatched neighbours of $w$ in $G_{\mathcal{P}, \mathcal{Q}}$ are connected to the other hatched vertices in $R$. 
2. Theorem. Let $\mathcal{P}$ and $\mathcal{Q}$ be additive induced-hereditary properties, $\mathcal{P} \circ \mathcal{Q} \neq \mathcal{O}^{2}$. Then $(\mathcal{P} \circ \mathcal{Q})$-recognition is NP-hard. Moreover, it is NP-complete iff $\mathcal{P}$ - and $\mathcal{Q}$-recognition are both in NP.

Proof: We will prove the first part. For the second part, one direction is easy, while the other follows from Theorem 1. Also by Theorem 1 (and by the well-known NP-hardness of recognising $\mathcal{O}^{3}[18]$ ), we need only consider the case where $\mathcal{P}$ and $\mathcal{Q}$ are irreducible. By Theorem 1 there is a strongly uniquely $(\mathcal{P}, \mathcal{Q})$-colourable graph $G_{\mathcal{P}, \mathcal{Q}}$ that we use to "force" vertices to be in $\mathcal{P}$ or $\mathcal{Q}$.

More formally, let the unique partition be $V\left(G_{\mathcal{P}, \mathcal{Q}}\right)=U_{\mathcal{P}} \cup U_{\mathcal{Q}}$. Choose $u \in U_{\mathcal{P}}$. If $G_{\mathcal{P}, \mathcal{Q}} \leq H$, and $v \notin V\left(G_{\mathcal{P}, \mathcal{Q}}\right)$ satisfies $N(v) \cap U_{\mathcal{Q}}=N(u) \cap U_{\mathcal{Q}}$, then in any $(\mathcal{P}, \mathcal{Q})$ colouring of $H, v$ must be in the $\mathcal{P}$-part ${ }^{3}$; otherwise, in $G_{\mathcal{P}, \mathcal{Q}}$ we could transfer $u$ over to the $\mathcal{Q}$ part, giving us a different $(\mathcal{P}, \mathcal{Q})$-colouring. Similarly we choose $w \in U_{\mathcal{Q}}$, whose neighbours we use to force vertices to be in $\mathcal{Q}$. $G_{\mathcal{P}, \mathcal{Q}}$ is our first gadget.

An end-block of a graph $G$ is a block of $G$ that contains at most one cut-vertex of $G$; in particular, if $G$ has no cut-vertices, then $G$ is itself an end-block. Let $B_{\mathcal{P}}$ be an end-block of $F_{\mathcal{P}} \in \mathcal{F}(\mathcal{P})$, chosen to have the least number of vertices among all the end-blocks of all the graphs in $\mathcal{F}(\mathcal{P})$ (see Figure 1). Because $\mathcal{P}$ is additive and non-trivial, $F_{\mathcal{P}}$ is connected and has at least two vertices, so $B_{\mathcal{P}}$ has $k \geq 2$ vertices. The point to note is that, if $H$ is a graph in $\mathcal{P}$, then adding an end-block with fewer than $k$ vertices produces another graph in $\mathcal{P}$.

Let $y_{\mathcal{P}}$ be the unique cut-vertex contained in $B_{\mathcal{P}}$ (if $B_{\mathcal{P}}=F_{\mathcal{P}}$, pick $y_{\mathcal{P}}$ arbitrarily), and let $x_{\mathcal{P}}$ be a vertex of $B_{\mathcal{P}}$ adjacent to $y_{\mathcal{P}}$. Let $F_{\mathcal{P}}^{\prime}$ be the graph obtained by adding an extra copy of $B_{\mathcal{P}}$ (incident to the same cut-vertex $y_{\mathcal{P}}$ ), and let $x_{\mathcal{P}}^{\prime}$ be a vertex in this new copy that is adjacent to $y_{\mathcal{P}}$.

Similarly, we choose $B_{\mathcal{Q}}$ to be an end-block of $F_{\mathcal{Q}} \in \mathcal{F}(\mathcal{Q})$, minimal among the endblocks of graphs in $\mathcal{F}(\mathcal{Q})$; we add a copy of $B_{\mathcal{Q}}$, and pick $x_{\mathcal{Q}}, y_{\mathcal{Q}}$ and $x_{\mathcal{Q}}^{\prime}$ as above. We identify $x_{\mathcal{P}}$ with $x_{\mathcal{Q}}, y_{\mathcal{P}}$ with $y_{\mathcal{Q}}, x_{\mathcal{P}}^{\prime}$ with $x_{\mathcal{Q}}^{\prime}$, and label the identified vertices $x, y, x^{\prime}$.

Finally, we force all the vertices of $F_{\mathcal{P}}^{\prime}$ (except for $x, y, x^{\prime}$ ) to be in $\mathcal{P}$, and all the vertices of $F_{\mathcal{Q}}^{\prime}$ (except for $x, y, x^{\prime}$ ) to be in $\mathcal{Q}$. That is, we add a copy of $G_{\mathcal{P}, \mathcal{Q}}$, and make every vertex of $F_{\mathcal{P}}^{\prime}-\left\{x, y, x^{\prime}\right\}$ adjacent to every vertex of $N(u) \cap U_{\mathcal{Q}}$, and every vertex of $F_{\mathcal{Q}}^{\prime}-\left\{x, y, x^{\prime}\right\}$ adjacent to every vertex of $N(w) \cap U_{\mathcal{P}}$ (cf. Figure 1 ).

It can be checked that the resulting gadget $R$ (for 'replicator') has the following properties:

Claim 1. In a $(\mathcal{P}, \mathcal{Q})$-colouring of $R$, if $x$ is in $\mathcal{P}$, then $y$ is in $\mathcal{Q}$ and $x^{\prime}$ is in $\mathcal{P}$; similarly, if $x$ is in $\mathcal{Q}$, then $y$ is in $\mathcal{P}$ and $x^{\prime}$ is in $\mathcal{Q}$. So $x$ and $x^{\prime}$ always have the same colour, that is different from that of $y$. Moreover, there is at least one colouring (in fact, exactly one) in which $x$ and $x^{\prime}$ are in $\mathcal{P}$, and at least one in which both are in $\mathcal{Q}$.

\footnotetext{
${ }^{3}$ To be precise, we mean that $v$ is coloured the same as $u$ : if $\mathcal{P}=\mathcal{Q}$ then a $(\mathcal{P}, \mathcal{Q})$-colouring is also a $(\mathcal{Q}, \mathcal{P})$-colouring, but we adopt the convention that the $\mathcal{P}$-part is the part containing $u$.
} 
Claim 2. Let $H$ be an arbitrary graph, and let $H_{R}$ be a graph obtained by identifying some vertex $z \in H$ with the vertex $x \in R$ (so this becomes a cut-vertex in $H_{R}$ ). Then a red-blue colouring of $H_{R}$ is a $(\mathcal{P}, \mathcal{Q})$-colouring iff it is a $(\mathcal{P}, \mathcal{Q})$-colouring of $H$ and a $(\mathcal{P}, \mathcal{Q})$-colouring of $R$.

Proof of Claim 2. The "only if" follows from the induced-heredity of $\mathcal{P}$ and $\mathcal{Q}$. For the converse we need to show, without loss of generality, that if every red component of $H$ and of $R$ is in $\mathcal{P}$, then every red component $C$ of $H_{R}$ is in $\mathcal{P}$. If $x \notin C$, then $C$ must be a red component of $H$ or of $R$.

If $x \in C$, then $C$ is formed from a red component $C_{H}$ of $H$ containing $z$, and a red component $C_{R}$ of $R$ containing $x$. Since $x$ is red, by Claim 1, $y$ is blue, so $C_{R} \subseteq B_{\mathcal{P}}-y_{\mathcal{P}}$. Now $B_{\mathcal{P}}$, on $k$ vertices, was a smallest possible end-block among the forbidden subgraphs for $\mathcal{P}$. Since $C_{H}$ is in $\mathcal{P}$, adding an end-block $C_{R}$ (or successively adding a sequence of end-blocks) on at most $k-1$ vertices produces another graph in $\mathcal{P}$.

We thus have a gadget that "replicates" the colour of $x$ on $x^{\prime}$, while preserving valid colourings.

Let $H_{\mathcal{P}}$ be a forbidden subgraph for $\mathcal{P}$ with the least possible number of vertices, say $p+1$; similarly choose $H_{\mathcal{Q}} \in \mathcal{F}(\mathcal{Q})$ on $q+1$ vertices, where $q+1$ is as small as possible, so any graph on at most $p$ (resp. $q$ ) vertices is in $\mathcal{P}$ (resp. $\mathcal{Q}$ ). Since $\mathcal{P}$ and $\mathcal{Q}$ are not both $\mathcal{O}, p+q \geq 3$, and so $p$-IN- $(p+q)$-COLOURING is NP-complete. We will construct a third gadget to transform this to $(\mathcal{P}, \mathcal{Q})$-colouring.

We start with an independent set $S$ on $p+q$ vertices, $\left\{x_{1}, \ldots, x_{p+q}\right\}$. For every $(p+1)$ subset of $S$, say $T_{j}=\left\{x_{1}, \ldots, x_{p+1}\right\}$, add a disjoint copy of $H_{\mathcal{P}}$ whose vertices are labeled $x_{1}^{j}, \ldots, x_{p+1}^{j}$. For each $i=1, \ldots, p+1$, use a new copy $R_{i, j}$ of $R$ to ensure that $x_{i}$ and $x_{i}^{j}$ are always coloured the same; to do this, identify the vertices $x$ and $x^{\prime}$ of $R_{i, j}$ with $x_{i}$ and $x_{i}^{j}$. For every $(q+1)$-subset of $S$ we add a copy of $H_{\mathcal{Q}}$ in the same manner. Thus every vertex $x_{i} \in S$ will have $\ell=\left(\begin{array}{c}p+q-1 \\ p\end{array}\right)+\left(\begin{array}{c}p+q-1 \\ q\end{array}\right)$ 'shadow vertices' $x_{i}^{1}, \ldots, x_{i}^{\ell}$ from copies of $H_{\mathcal{P}}$ and $H_{\mathcal{Q}}$. Call this gadget $N$ (for 'pin cushion' - the copies of $H_{\mathcal{P}}$ and $H_{\mathcal{Q}}$ being stuck into the independent set $S$ by 'pins' or 'replicators').

In a $(\mathcal{P}, \mathcal{Q})$-colouring of $N$, no $p+1$ vertices of $S$ can be in $\mathcal{P}$, and no $q+1$ vertices can be in $\mathcal{Q}$, so exactly $p$ vertices of $S$ are in $\mathcal{P}$, and exactly $q$ are in $\mathcal{Q}$. Conversely, suppose that exactly $p$ vertices of $S$ are coloured red, and the other $q$ are blue; colour each vertex $x_{i}^{j}$ the same as $x_{i}, 1 \leq i \leq p+q, 1 \leq j \leq \ell$. Then each copy of $H_{\mathcal{P}}$ has at most $p$ red and at most $q$ blue vertices, giving it a valid $(\mathcal{P}, \mathcal{Q})$-colouring. The colouring on the rest of each gadget $R_{i, j}$ is then forced, and we have a $(\mathcal{P}, \mathcal{Q})$-colouring of all of $N$.

Now, given a $(p+q)$-uniform hypergraph $\mathcal{H}$, we stick a copy of $N$ onto every hyperedge. The resulting graph is $(\mathcal{P}, \mathcal{Q})$-colourable iff $\mathcal{H}$ has a $p$-IN- $(p+q)$-COLOURING. 


\section{New directions}

How far can the main result be extended? Uniquely $\left(\mathcal{P}_{1}, \ldots, \mathcal{P}_{n}\right)$-partitionable graphs exist even in many cases where the $\mathcal{P}_{i}$ 's are not additive [12]; however, this includes finite $\mathcal{P}_{i}$ 's, so the existence of uniquely colourable graphs does not guarantee NP-hardness.

It may be useful to restate the result as follows: if the graphs in $\mathcal{F}(\mathcal{P})$ and $\mathcal{F}(\mathcal{Q})$ are all connected, then $(\mathcal{P}, \mathcal{Q})$-colouring is NP-hard. This is also true if the graphs in $\mathcal{F}(\mathcal{P})$ and $\mathcal{F}(\mathcal{Q})$ are all disconnected, since $G \in \mathcal{P} \circ \mathcal{Q} \Leftrightarrow \bar{G} \in \overline{\mathcal{P}} \circ \overline{\mathcal{Q}}$, where $\overline{\mathcal{P}}$ is defined by $\mathcal{F}(\overline{\mathcal{P}}):=\{\bar{H} \mid H \in \mathcal{F}(\mathcal{P})\}$.

A natural problem to tackle next would be classifying the complexity of $\mathcal{R}^{k}$-recognition, where $\mathcal{R}$ has both connected and disconnected minimal forbidden induced-subgraphs. One of the simplest such cases is $\mathcal{R}=(\mathcal{O} \cup \mathcal{K})$, where $\mathcal{K}$ is the set of all cliques: $\mathcal{F}(\mathcal{O} \cup \mathcal{K})=\left\{P_{3}, \overline{P_{3}}\right\}$. Gimbel et al. [16] noted that $G \in \mathcal{O}^{k} \Leftrightarrow n G \in(\mathcal{O} \cup \mathcal{K})^{k}$ (where $n=|V(G)|)$; so $(\mathcal{O} \cup \mathcal{K})^{k}$-recognition is NP-complete for $k \geq 3$ (and, in fact, polynomial for $k=1,2)$.

Another natural problem is $(\mathcal{P}, \mathcal{Q})$-colouring, where all graphs in $\mathcal{F}(\mathcal{P})$ are connected, and all those in $\mathcal{F}(\mathcal{Q})$ are disconnected. In all problems, it may make sense to restrict attention to hereditary properties with finitely many forbidden subgraphs.

Another class of problems often considered in the literature is $(\mathcal{D}: \mathcal{P})$-recognition: given a graph $G$ in the domain $\mathcal{D}$, is $G$ in $\mathcal{P}$ ? This is just $(\mathcal{D} \cap \mathcal{P})$-recognition; if $\mathcal{D}$ and $\mathcal{P}$ are both additive induced-hereditary, then so is $\mathcal{D} \cap \mathcal{P}$, with $\mathcal{F}(\mathcal{D} \cap \mathcal{P})=$ $\min _{\leq}(\mathcal{F}(\mathcal{D}) \cup \mathcal{F}(\mathcal{P}))$. We leave it as an open question, for reducible $\mathcal{P}$, to determine when $\mathcal{D} \cap \mathcal{P}$ is also reducible; Mihók's characterisations [20,21] of reducibility may be useful in finding an answer.

\section{Notes and acknowledgements}

The most important part of the proof is the 'replicator' gadget. Phelps and Rödl [23, Thm. 6.2] and Brown [7, Thm. 2.3] used different gadgets to perform similar roles. The forcing technique of Theorem 1 was first used in [19, Thm. 2] and [5, Lemma 3].

Contacts with Lozin were very helpful, as they spurred the author to look at $\left(K_{m}\right.$-free, $K_{n}$-free)-colouring, not knowing it had been settled in [9]. Kratochvíl and Schiermeyer [19] proved a special case of Theorem 2 that covered the case $m=2 ;\left(K_{2}\right.$-free, $K_{n}$-free)colouring; I started my proof for general $m$ and $n$ by adapting theirs, and ended up strengthening and simplifying it considerably.

I would like to thank Bruce Richter for many helpful conversations, detailed comments that improved the presentation of the paper, and for spotting a flaw in my original "pin cushion' gadget. The result here forms part of the Ph.D. thesis that I am writing under his supervision. I would also like to thank the Canadian government for fully funding my studies through a Commonwealth Scholarship. 


\section{References}

[1] D. Achlioptas, The complexity of G-free colourability, Discrete Math. 165-166 (1997) 21-30.

[2] M.O. Albertson, R.E. Jamison, S.T. Hedetniemi, S.C. Locke, The subchromatic number of a graph, Discrete Math 74 (1989) 33-49.

[3] A.J. Berger, Minimal forbidden subgraphs of reducible graph properties, Discuss. Math. Graph Theory 21 (2001) 111-117.

[4] A. Brandstädt, V.B. Le, T. Szymcak, The complexity of some problems related to Graph 3-COlOrability, Disc. Appl. Math. 89 (1998) 59-73.

[5] I. Broere and J. Bucko, Divisibility in additive hereditary properties and uniquely partitionable graphs, Tatra Mt. Math. Publ. 18 (1999) 79-87.

[6] J.I. Brown, A theory of generalized graph colourings, Ph. D. Thesis, Department of Mathematics, University of Toronto (1987).

[7] J.I. Brown, The complexity of generalized graph colorings, Discrete Appl. Math. 69 (1996) 257-270.

[8] J.I. Brown and D.G. Corneil, Perfect colourings, Ars Combin. 30 (1990) 141-159.

[9] L. Cai and D.G. Corneil, A generalization of perfect graphs - $i$-perfect graphs, $J$. Graph Theory 23 (1996) 87-103.

[10] A. Farrugia and R.B. Richter, Unique factorisation of additive induced-hereditary properties, to appear in Discuss. Math. Graph Theory.

[11] A. Farrugia and R.B. Richter, Factorisation, reducibility, co-primality, and uniquely colourable graphs, in preparation.

[12] A. Farrugia and R.B. Richter, Unique factorisation of induced-hereditary disjoint compositive properties, Research Report CORR 2002-ZZ (2002) Department of Combinatorics and Optimization,

[13] J. Fiala, K. Jansen, V.B. Le and E. Seidel, Graph subcolorings: complexity and algorithms, Lecture Notes in Computer Science 2204 (Proceedings, Boltenhagen, 2001) 154-165.

[14] M.R. Garey and D.S. Johnson, Computers and Intractability (W.H. Freman, New York, 1979).

[15] M.R. Garey, D.S. Johnson and L. Stockmeyer, Some simplified NP-complete problems, Theor. Comput. Sci. 1 (1976) 237-267. 
[16] J. Gimbel, D. Kratsch and L. Stewart, On cocolourings and cochromatic numbers of graphs, Disc. Appl. Math. 48 (1994) 111-127.

[17] S.L. Hakimi and E.F. Schmeichel, A note on the vertex arboricity of a graph, SIAM J. Discrete Math. 2 (1989) 64-67.

[18] R.M. Karp, Reducibility among combinatorial problems, in R.E. Miller and J.W. Thatcher (eds.), Complexity of computer computations, Plenum Press, New York, 85-103.

[19] J. Kratochvíl and I. Schiermeyer, On the computational complexity of $(O, P)$ partition problems, Discuss. Math. Graph Theory 17 (1997) 253-258.

[20] P. Mihók, G. Semanišin and R. Vasky, Additive and hereditary properties of graphs are uniquely factorizable into irreducible factors, J. Graph Theory 33 (2000) 44-53.

[21] P. Mihók, Unique Factorization Theorem, Discuss. Math. Graph Theory 20 (2000) $143-153$.

[22] B. Monien, correspondence with Brandstädt, Le and Szymcak, 1984.

[23] K.T. Phelps and V. Rödl, Algorithmic complexity of coloring simple hypergraphs and Steiner triple systems, Combinatorica 4 (1984) 79-88.

[24] T.J. Schaefer, The complexity of satisfiability problems, Proc. 10th Ann. ACM Symp. on Theory of Computing, Association for Computing Machinery, New York (1978) $216-226$.

[25] D.B. West, Introduction to graph theory, second edition, Prentice Hall, 2001. 